\title{
Atomic Resolution Single Walled Carbon Nanotube Nucleation Steps on Faceted Catalyst Particle Reveal Potential for Chirality Control
}

\author{
Pin Ann Lin ${ }^{1,2}$, Matthieu Picher ${ }^{1,2}$, Jose Leonardo Gomez Ballesteros ${ }^{3}$, Perla Balbuena ${ }^{3}$, and Renu \\ Sharma ${ }^{1}$ \\ ${ }^{1}$ Center for Nanoscale Science and Technology, National Institute of Standards and Technology, \\ Gaithersburg, MD 20899-6203, USA \\ ${ }^{2}$ University of Maryland - IREAP, College Park, MD 20742, USA \\ ${ }^{3}$ Department of Chemistry, Texas A\&M University, TX
}

Single-walled carbon nanotubes (SWCNTs) continue to be one of the most desirable materials for numerous nanotechnology applications due to their unique combination of quantum confinement and large surface area-to-volume ratio. Despite this, only limited numbers of SWCNT-based technologies have come to the market because of the difficulty in generating sufficient quantities of SWNCTs with uniform electronic properties. This is because the electronic properties of SWCNTs vary with their diameter, defect density and, most importantly, with their chirality. Although a few reports indicate that a degree of chirality control is possible with specific nanoparticle (NP) catalyst-support system [1,2], there is a fundamental lack of knowledge about what processes determine SWCNT chirality, particularly concerning the NT nucleation process on the catalyst, which precludes the design of efficient experimental approaches and rational catalyst design. We have employed an Environmental Scanning Transmission Electron Microscope (ESTEM) [3], operated at $300 \mathrm{kV}$ with an aberration corrector, which permits us to record real-time atomic resolution videos $\left(6\right.$ frames $\left.\mathrm{s}^{-1}\right)$ to capture nanotube cap nucleation, lift-off, and growth in $\mathrm{C}_{2} \mathrm{H}_{2}$ flowing over the $\mathrm{Co}-\mathrm{Mo} / \mathrm{MgO}$ system at synthesis temperatures. Detailed observations at this relatively slow frame rate are made possible by using lowpressure $\left(0.005 \mathrm{~Pa}\right.$ of $\left.\mathrm{C}_{2} \mathrm{H}_{2}\right)$ growth conditions (at $\left.650^{\circ} \mathrm{C}\right)$ to slow down the NT nucleation and growth rate.

Figure 1 shows a sequence of high-resolution images of an individual NP prior to SWCNT nucleation. Measurements of the Fast Fourier Transform (FFT) of the high-resolution images show that the structure of the NP converted from $\mathrm{CoO}$ to $\mathrm{Co}_{3} \mathrm{C}$, and then $\mathrm{Co}_{2} \mathrm{C}$, a carbon-rich phase (Fig 1a, b). Figure 2 shows a series of high-resolution images of an individual NP extracted from a video. The two surfaces, almost perpendicular to the support and external surface almost parallel to the support, of the $\mathrm{Co}_{2} \mathrm{C} \mathrm{NP}$ were identified as Co-terminated surface (020) and C+Co-terminated surface (210), respectively. A segment of graphene was first observed to nucleate on the (020) surface and then spread over the corner of the NP to the (210) surface (Fig. 2a). The growing structure expanded laterally until it reached the other (020) surface (Fig. b-d). The structure appears like a hemispherical cap, with the cap rim adhered well on both $\{020\}$ surfaces and the cap crown slightly lifted from the (210) surface (Fig. d). Continued addition of carbon from acetylene resulted in vertical growth to form a tube (Fig. e, f).

The final cap geometry comprises a graphene sheet that is attached to the pure metal terminated surfaces but that has lifted off from the carbon-metal terminated surface. The cap then guides the subsequent growth of the SWCNT. In addition to the metal-graphene interfacial energies, other factors, such as a preferential adsorption of carbon at low-coordination metal sites, higher diffusion rates on specific metal surfaces or subsurfaces, are involved in SWCNT nucleation and growth. Our observations indicate that tuning the orientation and size of the catalyst particle surfaces can significantly improve chiral selection 
of SWCNTs. More details of the mechanism of SWCNT nucleation and growth, and chirality control will be presented.

\section{References}

[1] W.H. Chiang et al. Nature Materials 2009, 8, 882

[2] M. He et al. Scientific Reports 2013, 3, 1460

[3] R. Sharma et al. Applied Physics Letters 2004, 84, 990
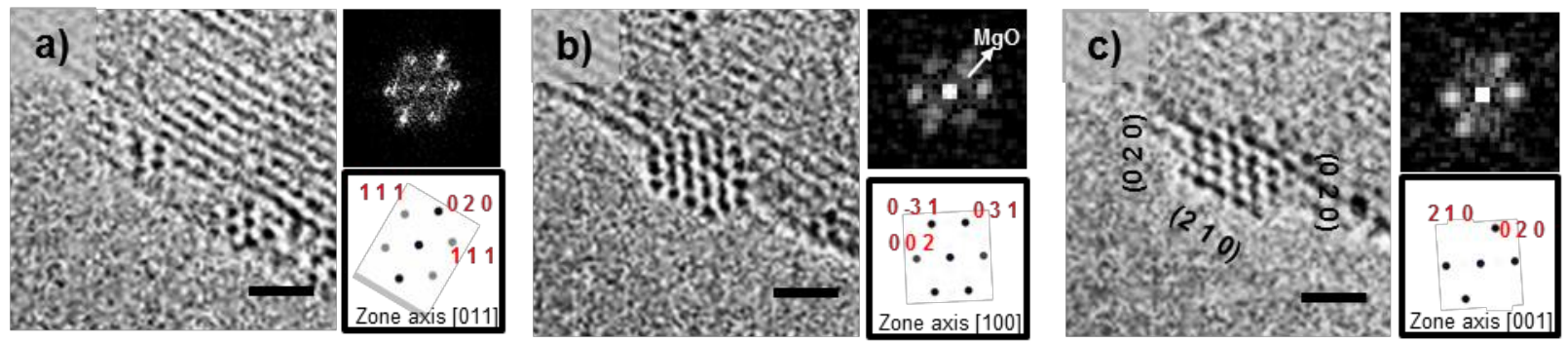

Figure 1. A sequence of NP catalyst structural changes prior SWCNT nucleation. An atomic-resolution image on the left panel with its corresponding selected area FFT image (top right panel) and calculated diffraction pattern (bottom right panel) is presented. The NP structure has been identified as a) $\mathrm{CoO}$ b) $\left.\mathrm{Co}_{3} \mathrm{C} \mathrm{c}\right) \mathrm{Co}_{2} \mathrm{C}$ and its surfaces (020) and (210) are shown. Scale bars are $1 \mathrm{~nm}$.
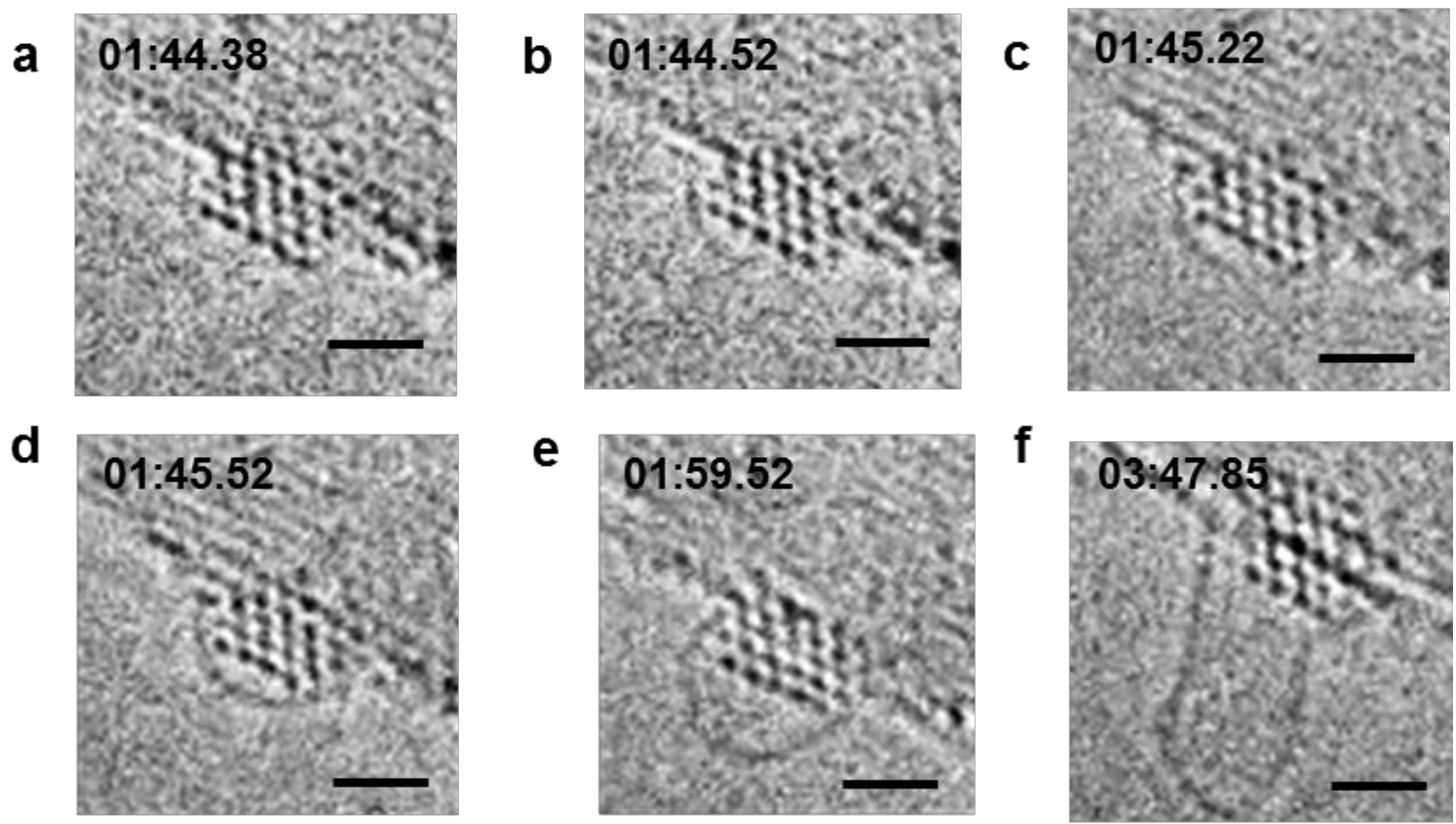

Figure 2. A series of atomic resolution images of a $\mathrm{Co}_{2} \mathrm{C}$-catalyzed SWNT growth. a,b,c,d) Steps of SWNT cap formation. e,f) after cap rim was adhered to both $\{020\}$ surfaces, lifted cap crown on (210) surface started to grow as a tube. Note that the orientation and surface combination of the NP remains the same throughout the SWNT nucleation and growth process. Scale bars are $1 \mathrm{~nm}$. 